\title{
A Study of Motor Bearing Fault Diagnosis using Modulation Signal Bispectrum Analysis of Motor Current Signals
}

\author{
Ahmed Alwodai ${ }^{1}$, Tie Wang ${ }^{2}$, Zhi Chen ${ }^{2}$, Fengshou Gu${ }^{1}$, Robert Cattley ${ }^{1}$, Andrew Ball ${ }^{1}$ \\ ${ }^{1}$ Centre for Efficiency and Performance Engineering, University of Huddersfield, Queensgate, Huddersfield HD1 3DH, UK; \\ ${ }^{2}$ Department of Vehicle Engineering, Taiyuan University of Technology Taiyuan, Shanxi Province, China \\ Email: F.gu@hud.ac.uk
}

Received April, 2013.

\begin{abstract}
Failure of induction motors are a large concern due to its influence over industrial production. Motor current signature analysis (MCSA) is common practice in industry to find motor faults. This paper presents a new approach to detection and diagnosis of motor bearing faults based on induction motor stator current analysis. Tests were performed with three bearing conditions: baseline, outer race fault and inner race fault. Because the signals associated with faults produce small modulations to supply component and high nose levels, a modulation signal bispectrum (MSB) is used in this paper to detect and diagnose different motor bearing defects. The results show that bearing faults can induced a detestable amplitude increases at its characteristic frequencies. MSB peaks show a clear difference at these frequencies whereas conventional power spectrum provides change evidences only at some of the frequencies. This shows that MSB has a better and reliable performance in extract small changes from the faulty bearing for fault detection and diagnosis. In addition, the study also show that current signals from motors with variable frequency drive controller have too much noise and it is unlikely to discriminate the small bearing fault component.
\end{abstract}

Keywords: Induction Motor; Motor Current Signature; Power Spectrum Bispectrum; Motor Bearing

\section{Introduction}

A general review of monitoring and fault diagnosis techniques are studied in $[1,2]$. The different faults in an electrical machine can be classified as follows [3]:

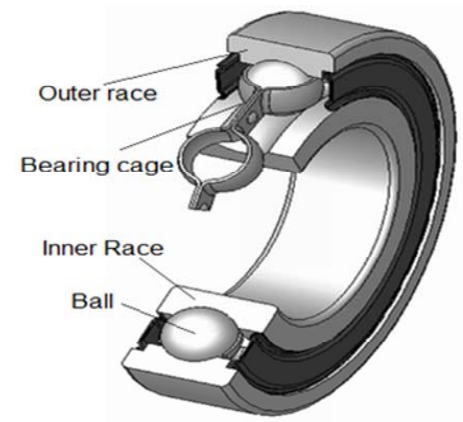

Figure 1. Components of the rolling ball bearing.

- Stator faults, for example, short circuit, loss of a supply phase.

- Rotor faults, for example, broken bar, broken endring.
- Static and dynamic eccentricities.

- Bearing faults.

Studies have shown that the common faults in induction motors (about $40 \%-50 \%$ ) happen in rolling bearings, depending on the type of installation, the motor size, and the supply voltage [4]. In general it is due to manufacturing faults, lack of lubrication, installation errors and wear and tear. According to the affected elements, shown in Figure 1, bearing faults can be classified as inner ring, outer ring, ball element and cage faults.

The inner ring is mounted on the shaft of the machine and is usually the rotating part whereas the outer ring is fixed in the housing of the machine and in most cases it does not rotate. The rolling elements may be balls, cylindrical rollers, spherical rollers, tapered rollers or needle rollers. They rotate against the inner and outer ring raceways and transmit the load acting on the bearing via small surface contacts separated by a thin lubricating film. The cage separates the rolling elements to prevent metal-to-metal contact between them during operation. Seals are important for protection of bearing from contamination and keep the lubricant inside the bearing sur- 
roundings.

\section{Bearing Fault Modes}

This paper considers rolling-element bearings with a geometry shown in Figure 2. The bearing consist essentially of the outer and inner raceways, the balls, and the cage, which keeps the distances between the balls equals. The number of balls is $\mathrm{N}_{\mathrm{b}}$, their diameter is $\mathrm{D}_{\mathrm{b}}$, and the pitch diameter is $D_{p}$. The point of contact between a ball and the raceway is characterized by the contact angle $\beta$.

Bearing faults can be classified into distributed and localized defects [5]. Distributed defects affect a whole region and are difficult to characterize by distinct frequencies. On the other hand, single-point defects are localized and can be classified according to the following affected element:

- Outer raceway defect

- Inner raceway defect

- Ball defect

- Cage defect

With each type of bearing fault, a characteristic frequency can be associated. This frequency is corresponding to the periodicity by which an irregularity appears due to the existence of the fault. The characteristic frequencies are functions of the bearing geometry and the mechanical rotor frequency $f_{r}$. For the four considered fault types, the characteristic frequency takes the following expressions[3, 5]:

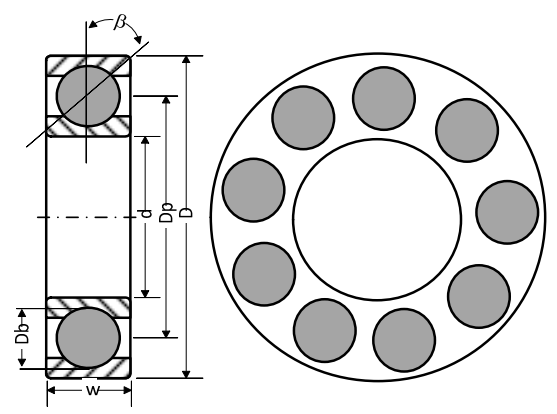

Figure 2. Geometry of a rolling-element bearing.

$$
\begin{gathered}
f_{o}=\frac{N_{\mathrm{b}}}{2} f_{\mathrm{r}}\left(1-\frac{\mathrm{D}_{\mathrm{b}}}{\mathrm{D}_{\mathrm{p}}} \cos \beta\right) \\
f_{i}=\frac{N_{b}}{2} f_{r}\left(1+\frac{D_{b}}{D_{p}} \cos \beta\right) \\
f_{b}=\frac{D_{p}}{D_{b}} f_{r}\left(1-\frac{D_{b}^{2}}{D_{p}^{2}} \cos ^{2} \beta\right) \\
f_{c}=\frac{1}{2} f_{r}\left(1-\frac{D_{b}}{D_{p}} \cos \beta\right)
\end{gathered}
$$

where $f_{o}$ is the outer race fault frequency, $f_{i}$ is the inner race fault frequency, $f_{b}$ is the ball fault frequency and $f_{c}$ is cage fault frequency.

The characteristic fault frequencies are the result of the absolute motion (vibration) of the machine. The stator current is not affected by the absolute motion of the machine, but rather by a relative motion between the stator and rotor (i.e., changes in the air gap). In the instance of a bearing fault, the characteristic fault frequencies are essentially modulated by the electrical supply frequency.

\section{Bearing Fault Detection by Stator Current Analysis}

The most often mentioned model studying the influence of bearing damage on the induction machine stator current was presented by Schoen etal [6]. The author considers the generation of rotating eccentricities at bearing fault characteristic frequencies $f_{v}$, which leads to periodical changes in the machine inductances. This should produce additional frequencies $f_{b f}$ in the stator current, which can be predicted by:

$$
f_{b f}=\left|f_{s} \pm k f_{v}\right|
$$

where $f_{s}$ is the electrical supply frequency, $f_{v}$ is one of the four characteristic fault frequencies defined by Equation 1 through Equation 4 .and $\mathrm{k}=1,2,3 \ldots$

This model has been used in several recent works [6-8]. In [6], two types of faults were tested, namely a hole drilled through the outer race and indentation produced in both the inner and outer surfaces. For both faults, the vibration and current spectra are analyzed in both loaded and unloaded motor cases. The first faulty condition (outer race defect)is characterized by $f_{o}$ and $2 f_{o}$ components in the vibration spectrum and $\left|f_{s} \pm f_{o}\right|$ and $\left|f_{s} \pm 2 f_{o}\right|$ com- ponents in the current spectrum. The second fault (inner race defect) is highlighted byf $f_{0}, 2 f_{o}$, and $f_{i}$ components in the vibration spectrum and $\left|f_{s} \pm f_{o}\right|,\left|f_{s} \pm 2 f_{o}\right|$, and $\left|f_{s} \pm f_{i}\right|$ components in the current spectrum. The authors claim that the characteristic fault frequency components are relatively small when compared to the rest of the current spectrum. The largest components occur at multiples of the supply frequency and are caused by saturation, winding error distribution, and supply voltage changes. However, an evaluation of the amplitude of these largest components in different cases (healthy and two types of fault condition) is not shown. In [7], two inner race faults (spalls and drilled hole) are analyzed, and the authors point out a problem related to the experimental simula- tion of bearing faults: The act of disassembling, re- mounting, and realigning the test motor can significantly alter the vibration and current spectra. The results show that, for both defects, the characteristic fault-frequency components are clearly visible only in the vibration spec- trum and not in the current spectrum. Both inner and outer raceway defects are analyzed in [8]. They show some differences in the amplitudes of the 
current spec- trum of a $1.5 \mathrm{~kW}$ induction motor at full load, but the characteristic fault-frequency components do not stand out clearly.

A new analytical model was suggested by [3] to take into account the load-torque variations caused by bearing faults in addition to the effect of relative motion between rotor and stator. In this way, new frequencies in the current spectrum are identified for the defects in the inner raceway.

$$
f_{i}^{\prime}=\left|f_{s} \pm f_{r} \pm k f_{i}\right|
$$

In [9] a three-phase 2.2-kW two-pole induction motor was used and the motor is equipped with two rolling ball bearings, type $6205 \mathrm{Z}$, with nine balls, lubricated with grease. The research presents four different types of bearing defects namely, crack in the outer race, hole in the outer race, deformation of the seal, and corrosion. The tests are performed under no-load and full-load.

The faults in the outer race have noticeable effects on the current spectrum, both in load and no-load conditions; the result shows a considerable increase of the third and seventh harmonic components at no load, and an increase of the even harmonics at high frequencies in the load condition. In case of no-load the second harmonic $(k=2)$ of the predicted frequency obtained by Equation. 5 exist on the other hand it does not appear under the load conditions studied.

In general previous studies have demonstrated the capability of current power spectrum based bearing fault signatures to detect bearing faults and produced diagnostic features of different bearing faults. However, because of high background noise levels of the current signals, especially when the motor is supplied by a variable speed drive (VFD), the features are often very weak.

This paper will examine the motor current spectra of healthy, outer race faults and inner race faults of an unloaded machine supplied with and without a VFD. To confirm the existences of the bearing characteristic frequencies, the motor is disconnected from the test rig and connected to an independent power supply directly to reduce the noise which is caused by the inverter and drive couplings. The current signals are analyzed by both power spectrum (PS) and modulation signal bispectrum (MSB), The latter analysis technique is a modified bispectrum analysis and has been demonstrated in previous studies to have good performance in noise reduction [13, 14] and is expected to produce more reliable bearing fault detection than that of power spectrum.

\section{Analysis Techniques}

\subsection{Power Spectrum}

The power spectrum method is generally used to describe the power distribution of the current signal in the fre- quency domain. Usually it is calculated using Fourier transform (FT) by:

$$
P(f)=E\left\{X(f) X^{*}(f)\right\}
$$

Where $X(f)$ and its conjugate $X^{*}(f)$ are the Fourier transform of the signal sequence $X(n)$, and $E\{$.$\} is$ the expectation operator.

\subsection{Conventional Bispectrum}

The bispectrum analysis is a type of higher order spectra, which is used by a large number of researchers since the 1980s [10] in different fields such as communications and medicine. Given a discrete time current signal $x(n)$, its discrete Fourier transform (DFT), $X(f)$ is defined to be

$$
X(f)=\sum_{k=-\infty}^{\infty} x(n) e^{-j 2 \pi f n}
$$

According to [11] the conventional bispectrum can be defined as

$$
B\left(f_{1}, f_{2}\right)=E\left\{X\left(f_{1}\right) X\left(f_{2}\right) X^{*}\left(f_{1}+f_{2}\right)\right\}
$$

Wheref1, $f 2$ and $f 1+f 2$ indicate the individual frequency components achieved from Fourier series integral.

Bispectrum analysis has a number of unique properties such as nonlinear system identification, phase information retention and Gaussian noise elimination when compared with power spectrum analysis. Especially, bispectrum is an effective tool for detecting quadratic phase coupling which occurs when two waves interact non-linearly and generate a third wave with a frequency and phase equal to the sum (or difference) of the first two waves.

A summary of the various bicoherence estimators can be found in [12]. The definition of squared bicoherence used in Equation 11 is chosen because it is bounded between 0 and 1 which is useful for comparing the degree of nonlinearity or coupling effect between different signals.

$$
b^{2}\left(f_{1}, f_{2}\right)=\frac{\left|B\left(f_{1}, f_{2}\right)\right|^{2}}{E\left\{\left|X\left(f_{1}\right) X\left(f_{2}\right)\right|^{2}\right\} E\left\{\left|X\left(f_{1}+f_{2}\right)\right|^{2}\right\}}
$$

A normalized form of the bispectrum or bicoherence is usually used to measure the degree of coupling between coupled components. The bicoherence is close to 1 if there are nonlinear interactions among frequency combinations $f_{1}, f_{2}$ and $f_{1}+f_{2}$. On the other hand, a value of near 0 means an absence of interactions between the components.

\subsection{Modulation Signal Bispectrum (MSB)}

Equation 10 includes only the presence of nonlinearity from the harmonically related frequency components: $f_{1}$, 
$f_{2}$ and $f_{1}+f_{2}$. It overlooks the possibility that the occurrence of $f_{1}-f_{2}$ might be due to the nonlinearity between $f_{1}$ and $f_{2}$ as well. Because of this, it is not adequate to describe amplitude modulation (AM) signals such as motor current signals.

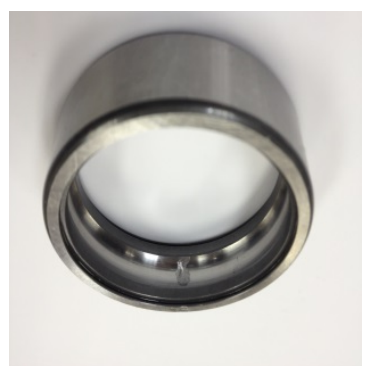

Figure 3. Outer race fault.

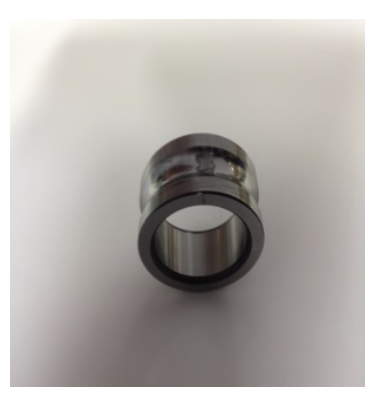

Figure 4. Inner race fault.

To improve the performance of the conventional bispectrum in characterizing the motor current signals, a new variant of the conventional bispectrum, named as a modulation signal bispectrum (MSB) is examined in [13, 14] as in Equation 12

$$
B_{M S}\left(f_{1}, f_{2}\right)=E\left\{X\left(f_{2}+f_{1}\right) X\left(f_{2}-f_{1}\right) X^{*}\left(f_{2}\right) X^{*}\left(f_{2}\right)\right\}
$$

Furthermore, to make a direct comparison with power spectrum in Equation 7 a normalized version of Equation 12 is introduced as:

$$
\begin{aligned}
& B_{M S N}\left(f_{1}, f_{2}\right) \\
& =E\left\{X\left(f_{2}+f_{1}\right) X\left(f_{2}-f_{1}\right) \frac{X^{*}\left(f_{2}\right)}{\left|X\left(f_{2}\right)\right|} \frac{X^{*}\left(f_{2}\right)}{\left|X\left(f_{2}\right)\right|}\right\}
\end{aligned}
$$

In Equation 13 the amplitude of $\frac{X^{*}\left(f_{2}\right)}{\left|X\left(f_{2}\right)\right|}$ which relates to carrier component $f_{2}$ is unity. Thus the amplitude of the modulation signals bispectral peaks are determined purely by the magnitude of the sideband components. In other words the resultant modulation signal bispectral magnitudes are independent of the amplitude of the carrier component at supply frequency and hence can be directly compared with that of power spectrum.

\subsection{Bearing Faults and Test Setup}

Two types of common bearing faults have been tested in this study. The first one, an abrasive wear in the outer race has been considered; which models that caused by friction between ball and outer race surfaces due to lack of lubrication as shown in Figure 3, while the second case of fault is an inner race defect as shown in Figure 4.

To analyze the current signals, a dataset is acquired in a motor rig under different simulated fault cases and operating conditions. Figure 5 shows the schematic diagram of the test facility employed to examine motor stator faults. The system consists of an induction motor, variable speed controller, supporting bearings, couplings and DC generator as a load. The test motor is a threephase induction motor with rated output power of $4 \mathrm{~kW}$ at $1420 \mathrm{rpm}$ (two-pole pairs). The motor has 28 rotor bars and 36 stator slots. Two rolling ball bearings, type $6206 Z Z$ deep groove ball bearing measuring 30x62×16 $\mathrm{mm}$, with nine balls $(\mathrm{Nb}=9), \mathrm{Db}=9.52 \mathrm{~mm}$ and $\mathrm{Dp}=46.0$ $\mathrm{mm}$, are used to support the rotor . To change the speed of the test motor, a digital variable speed controller is attached to the test rig between the power line source and the motor. The controller can be programmed to any specific shaft rotation speed between 0 and 1500rpm. The induction motor is directly coupled with a DC generator. The field of the generator is connected to a DC source through a controller while the generated power was fed back to the mains electrical grid via an inverter and the load on the induction motor can be adjusted by changing the field resistance of the DC generator. The operating load can be varied from no load to full load via the control panel.

A power supply measurement unit was designed to measure the instantaneous $\mathrm{AC}$ voltages, currents and power, using hall-effect voltage and current transducers and a universal power cell by the University staff. A shaft encoder, mounted on the shaft end produces 100 pulses per revolution for measuring the motor speed. A piezoelectric accelerometer, mounted on the bearing end-caps, is used to measure vibration of the motor. The accelerometer has a sensitivity of $5.0 \mathrm{mV} / \mathrm{ms} 2$ with a frequency range from 0.5 to $5000 \mathrm{~Hz}$, the current transducer has a sensitivity of $0.1 \mathrm{~A} / \mathrm{V}$ and a measurement range of 50A which allows the small changes of current and vibration to be measured with adequate accuracy.

During the tests the data was acquired using a GST YE6232B high speed data acquisition system. This system has 16 channels, each with 24 bit resolution, a maximum sampling frequency of $96 \mathrm{kHz}$, which allows the details of the $50 \mathrm{~Hz}$ component, the high order supply harmonics, rotor bar pass frequency, stator bar pass frequency and motor bearing frequency to be recorded for further analysis. 


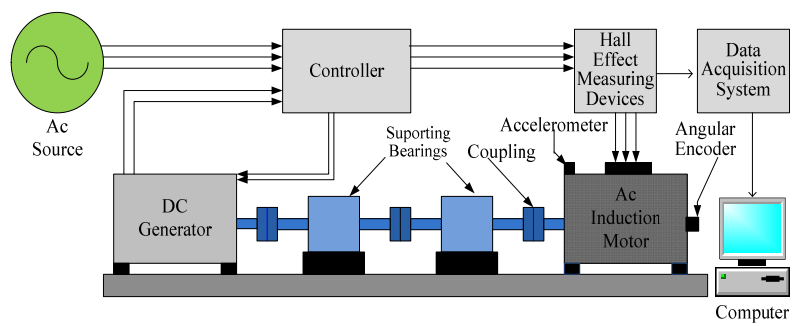

Figure 5. Schematic of induction motor test rig.

To evaluate the performance of PS and MSB analysis, current signals were collected under three different motor conditions: healthy motor, outer race fault and inner race fault and under four successive load conditions: zero, $25 \%, 50 \%$ and $75 \%$ of full load, which allows the diagnostic performance to be examined at different loads and avoid any possible damage to the test system at the full load when the faults are simulated. However, because of noises which are caused by controller, the characteristic bearing fault frequencies were difficult to differentiate from complex spectrum patterns. The motor was then disconnected from the loading system and the test was carried out with and without the controller and the results will be discussed in next section.

In the meantime vibration signals were also measured under healthy and two bearing faults to confirm the effectiveness of fault induced based on the vibration frequencies values which calculated by Equation 1 to Equation 4 .

\section{Results and Discussions}

The dataset is processed off-line using a Matlab program which implements PS and MSB calculations simultaneously with a 4-term Blackman-Harris window and 100 times of average. In this way they can be compared directly in the performance of identifying the characteristic frequencies and quantifying their spectral peaks.

\subsection{Effect of Controller}

The phase current signal is firstly examined under different supply cases (with and without controller). Before exploring the current spectrum, vibration signals are examined to confirm the introduction of the faults.

Figure 6 shows the vibration spectrum for both the outer race and inner race faults. The characteristic frequency and their harmonics are clearly shown. In addition, the amplitude at the fundamental frequency $f_{i}$ is higher than that of $f_{o}$, indicating that the fault from inner race may be higher than that of the outer race. These then shows that the two types of faults have been induced with sufficient severity so that the vibration based method can detect them.

Figure 7 shows a comparison of current spectrum be- tween a healthy case and two faulty bearings under four different loads with the VFD controller. To highlight the small components, the $\mathrm{Y}$-scale is fixed to $0.4 \mathrm{~A}$. It is difficult to find the spectrum components at the characteristic frequencies suggested in Equation 5 due to bearing faults for all load conditions. Instead there are clear peaks at frequencies $(f s \pm f r)$ which become higher with faults. In addition the peak values decreases with the increase of load. These changes show that there is noticeable eccentricity which may be induced to the rotor system when refitting the faulty bearings. It may indicate that the eccentricity induced changes mask the changes due to bearing faults. In addition, the controller will cause high level of noise because VFD provides a pulse width modulated signal source, which may also mask the small component at bearing characteristic frequencies.

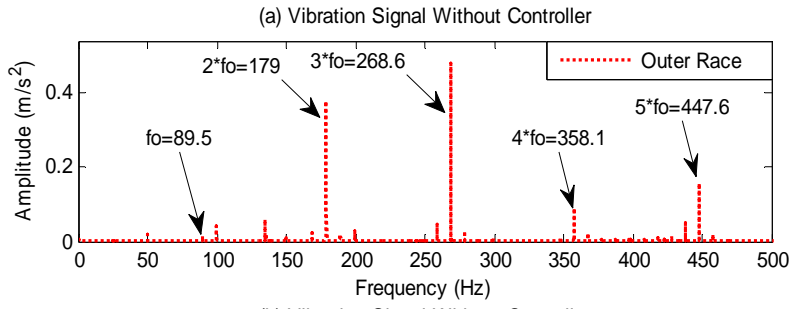

(b) Vibration Signal Without Controller

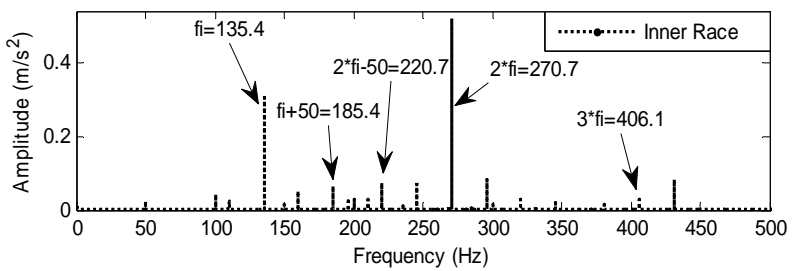

Figure 6. Spectrum of vibration for faulty bearings.
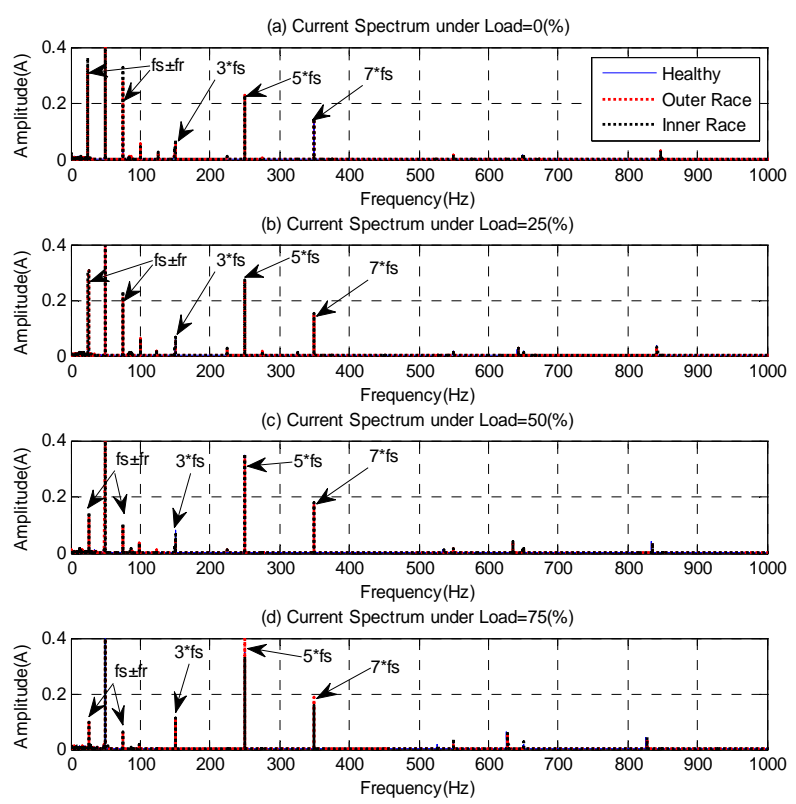

Figure 7. Current spectra with controller. 
To eliminate these two types of noise and other possible influences caused by downstream components such as generators and couplings, the motor was disconnected from the control system and connected directly to main power supply. The unloaded machine was then tested under three corresponding conditions, namely, healthy, outer race and inner race faults. Figure 8 presents the spectrum of the phase current from the unloaded motor with and without the controller.

The spectrum with controller shown in Figure 8(a) has very rich frequency components which include not only the harmonics of supply frequency but also distinctive components at $100 \mathrm{~Hz}, 200 \mathrm{~Hz}$ etc. The later indicates certain degree of the imbalance of the supply system. Moreover, the background noise level is more than $40 \mathrm{~dB}$ higher than that of the case without controller. This shows that it is likely the bearing fault frequency is masked by the background noise. Therefore, further study of current signature analysis is carried out under the condition of no-controller and no-load. Considering the small components and complicated spectrum pattern due to bearing faults and high order harmonics, more advanced MSB analysis along with PS analysis will be used to process the measured signals.

\subsection{Outer Race Fault Detection and Diagnosis}

Figure 9 shows the results of MSB and PS analysis for both the healthy and faulty outer race cases in the frequency ranges around the first two harmonics of bearing fault frequency. For the healthy case both types of spectra do not show significant peaks at the characteristic frequencies of $f_{s} \pm f_{o}$ and $f_{s} \pm 2 f_{o}$ forPS or $f_{o}$ and $2 f_{o}$ for MSB. For the faulty case clear peaks can be observed at some of these frequencies. MSB shows distinctive peaks at both $f_{o}$ and $2 f_{o}$. However, from PS spectra only one spectral peak at $f_{s}-2 f_{o}$ can be discriminated in Figure 9(b2) and the rest of three peaks cannot be determined due to high background noise level. This shows that MSB have better capability to separate the small bearing features from noisy measurement.

To show more details of the spectrum changes, Table 1 summarizes the peak values at the characteristic frequencies of interest. For comparison the peak values at corresponding frequency are also listed even if they are not very distinctive in the spectrum. The table shows the peaks for the faulty one are higher than that of the healthy one, showing that faulty bearings cause more fluctuation in the current signal, which is consistent with that predicted in theory.

Moreover, MSB peaks show higher percentage increases at both of the peaks compared with that of PS. This demonstrates that MSB has a better diagnosis capability. Especially, it shows that the peak increase at $2 f o$ is higher than atfo, which shows is agree with the the spectrum results of vibration shown in Figure 6(a) in that the fault induce higher amplitude in the second harmonic component.
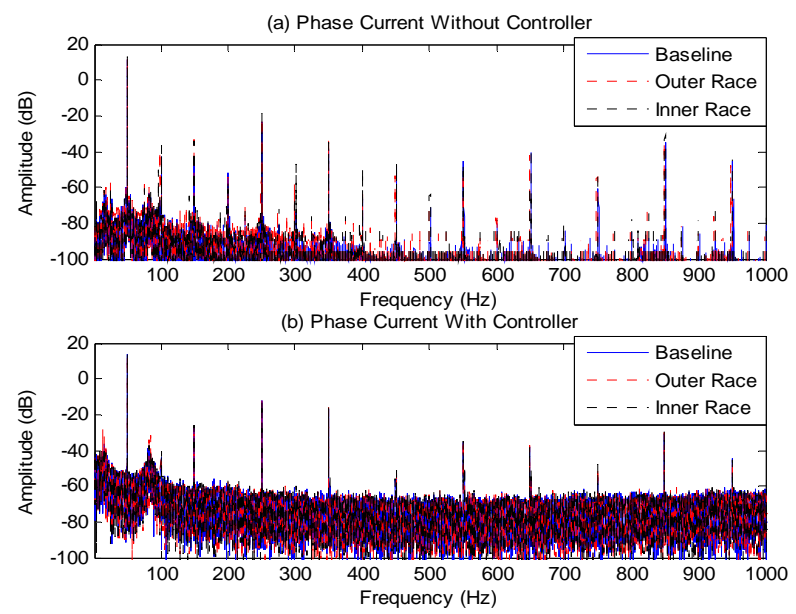

Figure 8. Current spectra with controller and without controller.

Table 1. Comparison of spectral peaks for outer race fault.

\begin{tabular}{ccccc}
\hline Spectral Peak & Healthy[1E-6] & Faulty[1E-6] & {$[1 \mathrm{E}-6]$} & {$[\%]$} \\
\hline MSB at $f o$ & 0.0007 & 0.0072 & 0.0065 & 916 \\
\hline PS at $f s-f o$ & 0.0182 & 0.1191 & 0.1009 & 553 \\
\hline PS at $f s+f o$ & 0.0032 & 0.0106 & 0.0074 & 231 \\
\hline MSB at $2 f_{0}$ & 0.00015 & 0.00345 & 0.00330 & 2154 \\
\hline PS at $f s-2 f o$ & 0.00342 & 0.02254 & 0.01912 & 559 \\
\hline PS at $f s+2 f o$ & 0.00082 & 0.00804 & 0.00722 & 877 \\
\hline
\end{tabular}

\subsection{Inner Race Fault Detection and Diagnosis}

Figure 10 shows the results of MSB and PS analysis for both the healthy and faulty inner race cases in the frequency ranges around the first two harmonics of bearing fault frequency. For the healthy case both types of spectra do not show significant peaks at the characteristic frequencies of $f s \pm f i$ and $f s \pm 2 f i$ for PS or fi and $2 f i$ for MSB. For the faulty case clear peaks can be observed at most of these frequencies. MSB shows distinctive peaks at both fi and $2 f i$,. Especially, the peak at $2 f i$ is quite distinctive as shown in Figure 10(b1). Without double, the presence of the inner race fault can be detected by MSB analysis. From PS spectra, three peaks can be determined at Figure 10 (a2), (b2) and (b3). However, the peak at $f s+$ fiin Figure10 (a3) cannot be resolved properly and has higher amplitude than that of healthy case. Because at least one set of sideband can be found it is possible now to detect the inner race fault by SP analysis. This 
also shows that PS is capable to detect the fault when the se- verity is higher which is confirmed by the vibration spectrum shown in Figure 6. In other words, PS is less reliable in detecting this inner race compared with MSB analysis.
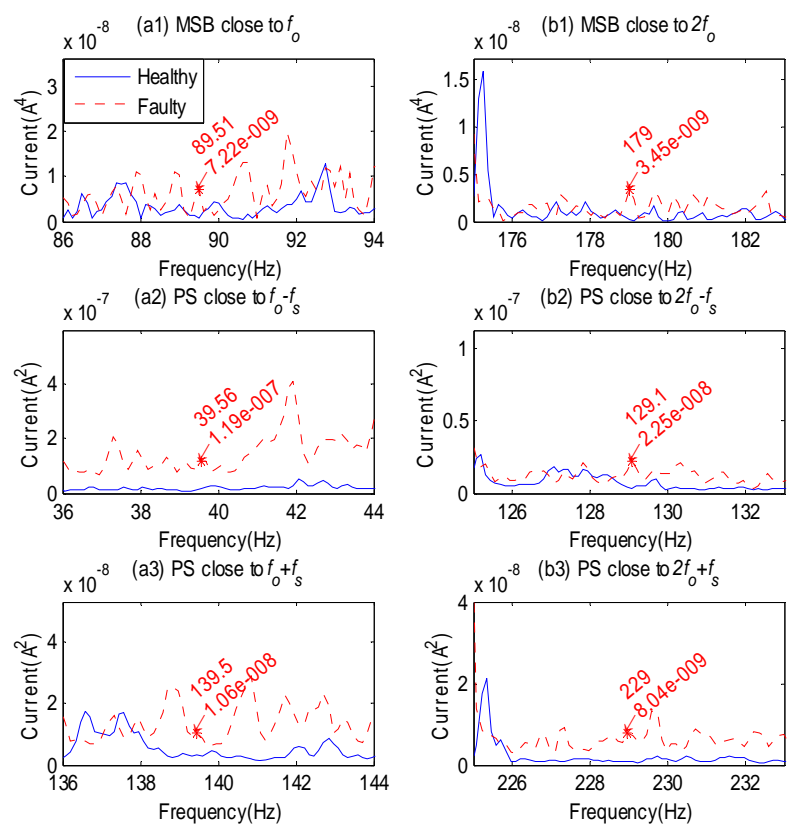

Figure 9. Spectrum in the frequency range close to $f_{o}$ and $2 f_{o}$.

To show more details of the spectrum changes, the peak values at characteristic frequencies of interest are also summarized, shown in Table 2. For comparison the peak value at $f_{s}+f_{i}$ is also listed even though it is not distinctive and has higher amplitude than the healthy case in the spectrum. The table shows that the peak values for the faulty one are higher than that of the healthy one except for the peak at $f_{s}+f_{i}$, showing that faulty bearings cause more fluctuation in the current signal, which is consistent with that predicted in theory.

Moreover, MSB peaks show higher percentage increases at both of the peaks compared with that of PS. This demonstrates that MSB has a better diagnosis capability. Especially, it shows that the peak increase at $2 \mathrm{fi}$ is higher than at fi, which is agree with the spectrum results of vibration shown in Figure6(b) in that the fault induce higher amplitude in the second harmonic component.

However, comparing the amplitude increases between Table 1 and Table 2 has found that the amplitudes of the inner race fault are smaller than that of outer race fault. This is not very consistent with that of vibration results show in Figure 6. However, this less amplitude changes may be due to the higher attenuation in the high frequency range of the induction motor, which can be understood in the spectra of Figure 8 in which the spectral amplitude has a monotonic decrease trend with the increasing of frequency. Based on this frequency response characteristics the amplitude changes at $f_{i}$, and its harmonics will be lower because $f_{i}$, is higher than $f_{o}$.

Table 2 Comparison of spectral peaks for inner race fault.

\begin{tabular}{ccccc}
\hline Spectral Peak & Healthy[1E-6] & Faulty[1E-6] & {$[1 \mathrm{E}-6]$} & {$[\%]$} \\
\hline MSB at fi & 0.0058 & 0.0174 & 0.0116 & 200 \\
\hline PS at fs- fi & 0.1075 & 0.6741 & 0.5666 & 527 \\
\hline PS at fs + fi & 0.0036 & 0.0021 & -0.0016 & -43 \\
\hline MSB at 2fi & 0.000294 & 0.001578 & 0.001284 & 437 \\
\hline PS at fs- 2fi & 0.000901 & 0.003153 & 0.002252 & 249 \\
\hline PS at fs+ 2fi & 0.000309 & 0.001685 & 0.001376 & 445 \\
\hline
\end{tabular}
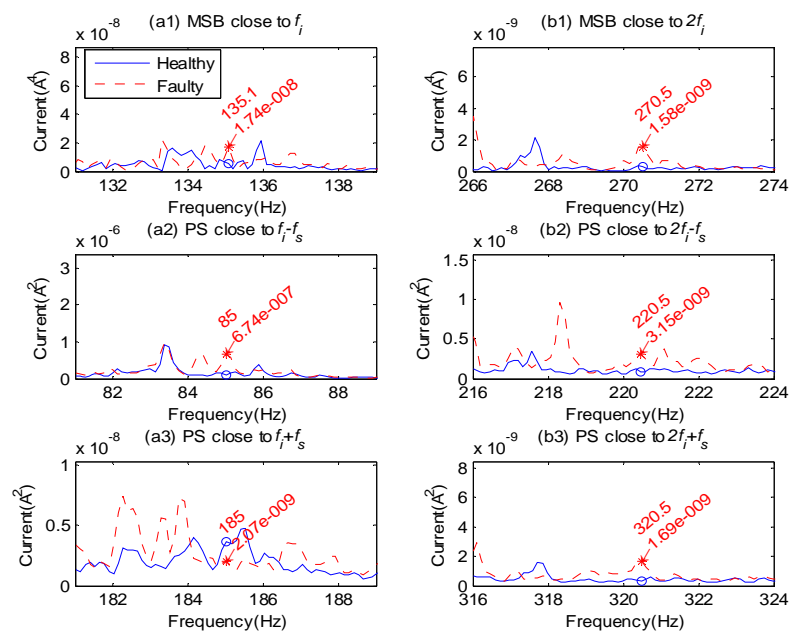

Figure 10. Spectrum in the frequency range close to $f_{i}$ and 2 $f_{i}$.

\section{Conclusions}

MSB analysis is evaluated using motor current signals to detect and diagnosis motor bearing faults. From its spectrum presentations, it can be found that MSB show a simpler spectrum structure compared with the power spectrum. It means that MSB shows peaks only at $k f_{o}$ and $k f_{i}$ for outer race and inner race faults respectively whereas power spectrum shows peaks at $\left(f_{s} \pm k f_{o}\right)$ and $\left(f_{s} \pm k f_{i}\right)$ which cause difficulty to be identified them when the spectrum components are rich. Moreover MSB pro- duces more accurate amplitude estimate due to its ca- pability of noise reduction and non-modulation compo- nent removal.

With MSB analysis the motor current can be used to detect and diagnosis bearing faults when the motor operates without VFD controller under no-load condition. The VFD induces too much noise into the system and it is not possible to discriminate the small bearing compo- 
nent for condition monitoring.

The bearing fault causes amplitude increases at the characteristic frequencies corresponding to outer race and inner race faults. MSB produces a reliable difference at these frequencies whereas PS only provides change evidences at some of the frequencies. This shows that MSB has a better performance in extract small changes from the faulty bearing for fault detection and diagnosis.

\section{REFERENCES}

[1] D. Basak, A. Tiwari and S. P. Das, "Fault Diagnosis and Condition Monitoring of Electrical Machines-A Review," in Proc. IEEE ICIT, 2006, pp. 3061-3066.

[2] M. E. H. Benbouzid, "A Review of Induction Motors Signature Analysis as a Medium for Faults Detection," IEEE Transactions Industrial Electron, Vol. 47, No. 5, 2000, pp. 984-993.doi:10.1109/41.873206

[3] M. Blödt, P. Granjon, B. Raison and G. Rostaing, "Models for Bearing Damage Detection in Induction Motors Using Stator Current Monitoring," IEEE Transactions On Industrial Electronics Vol. 55, No. 4, 2008, pp. 1813-1822.doi:10.1109/TIE.2008.917108

[4] A. Shrivastava and S. Wadhwani, "Condition Monitoring for Inner Raceway Fault of Induction Motor Ball Bearings,"International Journal of Electrical Engineering, Vol. 5, 2012, pp. 239-244.

[5] J. Stack, T. G. Habetler and R. G. Harley, "Fault ClassiFication and Fault Signature Production for Rolling Element Bearings in Electric Machines," IEEE Transactions Industrial Applications, Vol. 40, 2004, PP. 735-739.

[6] R. R. Schoen, T. G. Habetler, F. Kamran and R. Bartheld, "Motor Bearing Damage Detection Using Stator Current Monitoring," IEEE Transactions Industrial Applications, Vol. 31, No. 6, 1995, pp. 1274-1279.

\section{doi: $10.1109 / 28.475697$}

[7] R. R. Obaid, T. G. Habetler and J. R. Stack, "Stator Current Analysis for Bearing Damage Detection in Induction Motors,"in Proc. SDEMPED,Atlanta, 2003, pp. 182-187.

[8] I. Rodríguez and R. Alves, "Bearing Damage Detection of the Induction Motors Using Current Analysis,"in Proc. TDC IEEE/PES Transmiss.Distrib. Conf. Expo.: Latin America, August 2006, pp. 1-5.

[9] L. Frosini and E. Bassi, "Stator Current and Motor Efficiency as Indicators for Different Types of Bearing Faults in Induction Motors," IEEE Transactions on Industrial electronics, Vol. 57, No. 1, 2010, pp. 244-251. doi:10.1109/TIE.2009.2026770

[10] G. C. Zhang, M. G. e, H. Tong, Y. Xu and R. Du, "Bispectral Analysis for on-line Monitoring of Stamping Operation,"Engineering Applications of Artificial Intelligence, Vol. 15, No. 1, 2002, pp. 97-104. doi:10.1016/S0952-1976(02)00007-6

[11] W. B. Collis, P. R. White and J. K. Hammond, "Higher-order Spectra: The Bispectrum and Trispectrum," Mechanical Systems and Signal Processing, Vol. 12, No. 3, 1998, pp. 375-394. doi:10.1006/mssp.1997.0145

[12] J. W. A. Fackrell, S. McLaughlin and P. R. White, "Bicoherence Estimation Using the Direct Method. Part 1: Theoretical considerations,"Applications. Signal Process, Vol. 3,1995, pp. 155-168.

[13] F. Gu, Y. Shao, N. Hu and A. D. Ball, "Electrical Motor Current Signal Analysis Using a Modified Bispectrum for Ault Diagnosis of Downstream Mechanical Equipment," Mechanical Systems and Signal Processing," Vol. 25, No. 1,2011, pp. 360-372. doi:10.1016/j.ymssp.2010.07.004

[14] A. Alwodai, X. Yuan, Y. Shao, F. Gu, and A. D. Ball, "Modulation Signal Bispectrum Analysis of Motor Current Signals for Stator Fault Diagnosis," Proceedings of the 18th ICAC, September 2012. 